\title{
Plasma neurofilament light chain as a potential biomarker in Charcot-Marie-
}

\section{Tooth disease}

Elina Millere ${ }^{1,2}$, Dmitrijs Rots ${ }^{3}$, Joel Simrén ${ }^{4,5}$, Nicholas J Ashton ${ }^{4,5}$, Einars Kupats ${ }^{6}$, Ieva Micule Viktorija Priedite $^{8}$, Natalja Kurjane ${ }^{9,10}$, Kaj Blennow ${ }^{4,5}$, Linda Gailite ${ }^{3}$, Henrik Zetterberg ${ }^{4,5,11,12}$, Viktorija Kenina ${ }^{9,13}$

${ }^{1}$ Department of Neurology and Neurosurgery, Children's Clinical University Hospital, Riga, LV1004, Latvia

${ }^{2}$ Department of Doctoral Studies, Riga Stradins University, Riga, LV-1007, Latvia

${ }^{3}$ Scientific Laboratory of Molecular Genetics, Riga Stradins University, Riga, LV-1007, Latvia

${ }^{4}$ Department of Psychiatry and Neurochemistry, Institute of Neuroscience and Physiology, The Sahlgrenska Academy, University of Gothenburg, Mölndal, Sweden

${ }^{5}$ Clinical Neurochemistry Laboratory, Sahlgrenska University Hospital, Mölndal, Sweden ${ }^{6}$ Department of Neurology, Riga East Clinical University Hospital, Riga, LV-1038, Latvia ${ }^{7}$ Clinic of Medical Genetics and Prenatal Diagnostics, Children's Clinical University Hospital, Riga, LV-1004, Latvia

${ }^{8}$ BIOCON Laboratory, Riga, LV-1003, Latvia

${ }^{9}$ Department of Biology and Microbiology, Riga Stradins University, Riga, LV-1007, Latvia

${ }^{10}$ Outpatient Service Centre, Pauls Stradins Clinical University Hospital, Riga, LV-1002, Latvia

${ }^{11}$ Department of Neurodegenerative Disease, UCL Queen Square Institute of Neurology, London, UK

${ }^{12}$ UK Dementia Research Institute, UCL, London, UK

${ }^{13}$ Rare Disease Centre, Riga East Clinical University Hospital, Riga, LV-1038, Latvia

Corresponding author: Viktorija Kenina MD, PhD, kenina.viktorija@gmail.com

Total word count of the manuscript: 4,264

Keywords: Polyneuropathy; genetic and inherited disorders

Disclosure of conflict of interest: The authors declare no conflict of interest relevant to the submitted study.

This article has been accepted for publication and undergone full peer review but has not been through the copyediting, typesetting, pagination and proofreading process, which may lead to differences between this version and the Version of Record. Please cite this article as doi: $\underline{10.1111 / \text { ENE.14689 }}$

This article is protected by copyright. All rights reserved 
Disclosure of sources of funding: $\mathrm{HZ}$ is a Wallenberg Scholar supported by grants from the Swedish Research Council (\#2018-02532), European Research Council (\#681712), Swedish State Support for Clinical Research (\#ALFGBG-720931), Alzheimer Drug Discovery Foundation, USA (\#201809-2016862) and UK Dementia Research Institute.

Data Availability Statement: The data that support the findings of this study are available from the corresponding author upon reasonable request.

This article is protected by copyright. All rights reserved 
DR. ELINA MILLERE (Orcid ID : 0000-0001-5387-122X)

Article type : Original Article

Background. Charcot-Marie-Tooth disease (CMT) is a chronic, slowly progressing disorder. The lack of specific disease progression biomarkers limits the execution of clinical trials. However, neurofilament light chain (NfL) has been suggested as a potential biomarker for peripheral nervous system disorders. Methods. Ninety-six CMT patients and 60 healthy controls were enrolled in the study. Disease severity assessment included clinical evaluation with CMT Neuropathy Score version 2 (CMTNSv2). Blood plasma NfL concentrations were measured using the single molecule array (Simoa) NfL assay. Results. The NfL concentration was significantly higher in the CMT patient group than in the controls $(\mathrm{p}<0.001)$. Of the CMT patients, ones with type CMTX1 had a higher NfL level than those in the two other analysed subgroups (CMT1A and other CMT types) ( $\mathrm{p}=0.0498)$. The NfL concentration had a significant but weak correlation with the CMTNSv2 ( $\left.\mathrm{r}_{\mathrm{s}}=0.25, \mathrm{p}=0.012\right)$. In one CMT patient with an extremely elevated NfL level, overlap with chronic inflammatory demyelinating polyneuropathy was suspected. ROC analysis showed that an NfL concentration of $8.9 \mathrm{pg} / \mathrm{mL}$ could be used to discriminate CMT patients from controls, with an area under the curve of 0.881 . Conclusions. Our study confirmed that the plasma NfL concentration is significantly higher in CMT patients than in controls. Plasma NfL concentration was found to significantly, albeit weakly, reflect the clinical severity of CMT. In the future, NfL may be used, either individually or collaboratively, as a biomarker in the clinical context of suspected CMT; however, several issues need to be addressed first.

\section{INTRODUCTION}

Charcot-Marie-Tooth disease (CMT) is a hereditary neuropathy characterised by chronic motor and sensory polyneuropathy. Globally, CMT is the most common inherited neuropathy with a prevalence of 1:2,500 and is also the most common hereditary neuromuscular disorder. 
It is genetically and clinically highly heterogeneous and at present no effective pharmacological treatments exist ${ }^{1}$.

Neurofilament is a major cytoskeletal protein of neurons and is found in both the central and peripheral nervous systems. Neurofilament chains maintain the shape and structure of neurons. An especially high concentration of neurofilament is present in neuronal axons and axonal damage or loss releases neurofilament into the blood and/or cerebrospinal fluid ${ }^{2}$. Consequently, this raises the possibility of using the concentration of different neurofilaments in clinical routine practice as a general biomarker for disorders involving axonal degeneration ${ }^{3,4}$. Indeed, the role and biomarker characteristics of neurofilament chains have already been explored in various diseases ${ }^{5-10}$, including acquired and inherited neuropathies such as CMT ${ }^{11,12}$ as well as in hereditary amyloid neuropathy related to TTR mutations ${ }^{13}$. CMT mostly manifests in the peripheral nervous system, suggesting that the level of neurofilament light chain (NfL) in plasma could reflect the extent of peripheral nerve damage.

CMT is a slowly progressing disease, which makes evaluating disease progression difficult, especially in clinical trials. Several CMT treatment clinical trials have failed to demonstrate any effect on the progression of the disease despite strong preliminary and/or pre-clinical data ${ }^{14-16}$. The lack of specific disease progression biomarkers limits the design and execution of these clinical intervention trials ${ }^{17}$. A recent study by Sandelius et al. ${ }^{12}$ has shown that the plasma NfL concentration in CMT patients is not only increased but also correlates with disease severity. These data suggest that it could be used as a measure of disease progression. However, the measurement of plasma NfL levels in CMT patients is still far from being implemented in practice and clinical trials because these findings have not been validated nor replicated. Additionally, standardisation of the detection method and reference values is necessary before widespread adoption of the $\mathrm{NfL}$ measurement as a CMT disease progression marker.

In this study, we evaluated the potential of the plasma level of NfL as a biomarker in CMT and validated the results of Sandelius et al. ${ }^{12}$ in a similar setting using the same NfL detection method and a large, highly phenotyped population-based cohort of Latvian CMT patients. Additionally, we discuss current limitations and possible aberrations.

\section{METHODS}

\section{Participant evaluation and blood sampling}


We recruited a large cohort of CMT patients from geneticists', neurologists' and paediatric neurologists' clinical practices. CMT was diagnosed based on either symptoms and clinical/neurophysiological examination or a positive genetic testing result following cascade testing for a known pathogenic variant in the family. Participants were recruited and systematically evaluated in an outpatient setting. Patients responded to an in-house sociodemographic questionnaire and were clinically and neurophysiologically evaluated using standardised tests for CMT patients: neurography, which was performed by a certified specialist according to the standard polyneuropathy protocol; scoring of severity, which was performed in accordance with CMT Neuropathy Score version 2 (CMTNSv2) ${ }^{18}$, the corresponding CMT Examination Score (CMTES) was also calculated.

As a control group, our study included healthy individuals without any known neurological diseases or neurological symptoms. Blood sampling and storage were conducted following a strict standard operating procedure. Briefly, blood samples from patients and controls were taken in outpatient setting by certified medical staff and processed within one hour. Blood was collected into EDTA-containing tubes and centrifuged at $20^{\circ} \mathrm{C}$ at $3,500 \mathrm{rpm}$ for 10 minutes. Plasma was then aliquoted and stored at $-20^{\circ} \mathrm{C}$.

For genetic analysis, DNA was isolated from peripheral blood with EDTA using the commercial kit innuPREP Blood DNA Mini Kit (Analytik Jena, Germany) according to the manufacturer's protocol. PMP22 gene copy number analysis was performed for all patients using the MLPA kit P405 (MRC-Holland, The Netherlands) according to the manufacturer's protocol. The software Coffalyser.Net was used to confirm the diagnosis of CMT1A in cases with PMP22 duplication. For individuals with a normal PMP22 copy number, the GJB1 gene was analysed using Sanger sequencing ${ }^{19}$. The obtained sequences were aligned to the GJBI gene reference sequence NG_008357.1 and identified variants were classified in accordance with the ACMG variant interpretation guidelines ${ }^{20}$.

\section{Plasma NfL level measurement}

Maintained at $-20^{\circ} \mathrm{C}$, all samples at the same time were sent to the Institute of Neuroscience and Physiology, The Sahlgrenska Academy at the University of Gothenburg for plasma NfL analysis. Time from taking blood sample till performing NfL assay varied between 3-4 months, however the samples were kept at $-20^{\circ} \mathrm{C}$ meanwhile. The NfL concentration in plasma was measured using the single molecule array (Simoa) NfL assay (Quanterix, USA). Samples were analysed blindly and in a random order. Calibrators were run in duplicate and obvious outlier calibrator replicates were masked before curve fitting. Samples were diluted 4-fold and run separately. Results were adjusted to compensate for the dilution. Two quality 
control (QC) levels were run in duplicate at the beginning and end of each run. For the QC sample with a concentration of $15.8 \mathrm{pg} / \mathrm{mL}$, repeatability was $5.2 \%$ and intermediate precision was $5.2 \%$. For the QC sample with a concentration of $50.0 \mathrm{pg} / \mathrm{mL}$, repeatability was $2.7 \%$ and intermediate precision was $3.2 \%$. The dynamic range was $1.9-1,800 \mathrm{pg} / \mathrm{mL}$. The lower limit of quantification was $1.9 \mathrm{pg} / \mathrm{mL}$.

\section{Statistical analysis}

The normality of the continuous data was assessed with histograms, Q-Q plots and the Shapiro-Wilk test. For normally distributed data, the t-test was used to compare means between groups, while the Mann-Whitney $U$ test was used for non-normally distributed data. Discrete data were compared using Pearson's chi-square test. The correlation between continuous data was assessed by Spearman's correlation coefficient. All calculations were performed using $\mathrm{R}$ v3.6.0 software ${ }^{21}$. The difference between correlation coefficients was assessed using Fisher's $\mathrm{Z}$ test, using the cocor package in $\mathrm{R}^{22}$. To assess the utility of NfL to discriminate controls from CMT patients, a receiver operator curve (ROC) was constructed and analysed using the $p R O C$ package in $\mathrm{R}^{23}$. The best threshold for the NfL level was determined using Youden's index, and the area under the curve (AUC) with 95\% confidence interval $(\mathrm{CI})$ was calculated by $p R O C$.

Following appropriate recommendations ${ }^{24,25}$, the single patient in our study with an $\mathrm{NfL}$ level below the level of detection was given a value corresponding to half of the detection limit.

\section{Standard protocol approval and patient consent}

The study was approved by the Central Medical Ethics Committee of Latvia (No. 3/18-0321). Written informed consent was obtained from all participants in the study. The data supporting the findings of this study are available on request from the corresponding author. They are not publicly available due to privacy/ethical restrictions.

\section{RESULTS}

This study recruited 96 CMT patients (52/96 CMT patients were from 19 families) and 60 healthy subjects (Table 1). The patient group was subdivided according to the genetic findings and patients with no known genetic mutation were classified as "Other CMT". There was no difference in age or sex between the patient and control groups $(\mathrm{p}=0.238$ and $\mathrm{p}=0.087$, respectively).

This article is protected by copyright. All rights reserved 
Table 1. Study participant characteristics

\begin{tabular}{|c|c|c|c|c|c|}
\hline $\begin{array}{c}\text { Study } \\
\text { participants }\end{array}$ & $\begin{array}{c}\text { Total } \\
\text { (female/ } \\
\text { male) }\end{array}$ & $\begin{array}{c}\text { Mean } \\
\text { age (SD) }\end{array}$ & $\begin{array}{c}\text { Median } \\
\text { NfL, pg/mL } \\
\text { (IQR) }\end{array}$ & $\begin{array}{c}\text { Median } \\
\text { CMTNS } \\
\text { (IQR) }\end{array}$ & $\begin{array}{c}\text { Median } \\
\text { CMTES } \\
\text { (IQR) }\end{array}$ \\
\hline CMT group & $\begin{array}{c}96 \\
(51 / 45)\end{array}$ & $\begin{array}{c}38.6 \\
(18.4)\end{array}$ & $12.5(7.9)$ & $10(10.0)$ & $7(6.5)$ \\
\hline CMT1A & $\begin{array}{c}43 \\
(24 / 19)\end{array}$ & $\begin{array}{c}36.7 \\
(16.3)\end{array}$ & $12.5(5.9)$ & $12(7.0)$ & $6(5.0)$ \\
\hline CMTX1 & $\begin{array}{c}10 \\
(5 / 5)\end{array}$ & $\begin{array}{c}35.5 \\
(17.7)\end{array}$ & $16.0(5.8)$ & $10(16.8)$ & $7.5(11.2)$ \\
\hline Other CMT & $\begin{array}{c}43 \\
(22 / 21)\end{array}$ & $\begin{array}{c}41.2 \\
(20.5)\end{array}$ & $11.8(9.2)$ & $9(10.5)$ & $7(9.0)$ \\
\hline $\begin{array}{l}\text { Control } \\
\text { group }\end{array}$ & $\begin{array}{c}60 \\
(41 / 19)\end{array}$ & $\begin{array}{c}35.7 \\
(11.8)\end{array}$ & $5.2(2.8)$ & $\overline{\mathrm{NA}}$ & $\overline{\mathrm{NA}}$ \\
\hline
\end{tabular}

There was no difference in the NfL levels between sexes in the CMT and control groups ( $\mathrm{p}=1.00$ and $\mathrm{p}=0.14$, respectively). However, the NfL level moderately correlated with age in the controls and CMT group $\left(\mathrm{r}_{\mathrm{s}}=0.42, \mathrm{p}=0.001\right.$ and $\mathrm{r}_{\mathrm{s}}=0.31, \mathrm{p}=0.002$, respectively) (Figure 1). Despite the fact that the control group showed a greater correlation between NfL level and age than the CMT group, there was no significant difference between the levels of correlation ( $\mathrm{z}=-0.81, \mathrm{p}=0.42)$.

Figure 1. Correlation of neurofilament light chain (NfL) level with age in the two study groups. 


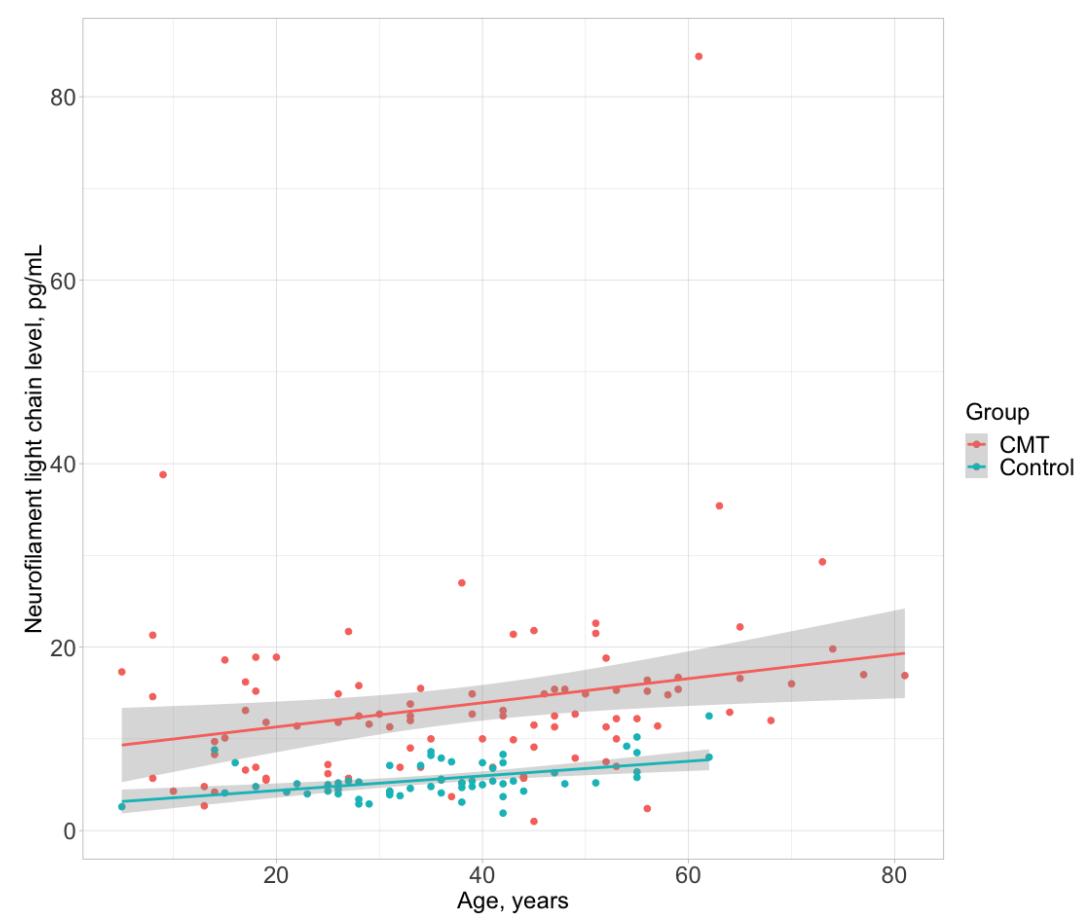

The plasma NfL concentration was significantly higher in the CMT group than in the controls $(\mathrm{p}<0.001)$. NfL concentrations measured in the study groups are shown in Figure 2 and the median plasma NfL level with IQR is shown in Table 1. Importantly, there was one noticeable outlier with an extremely high $\mathrm{NfL}$ level among the patients ( $\mathrm{NfL}=84.4$ vs. median=12.5 pg/mL).

Figure 2. Plasma neurofilament light chain (NfL) concentrations in the different study groups.

This article is protected by copyright. All rights reserved 


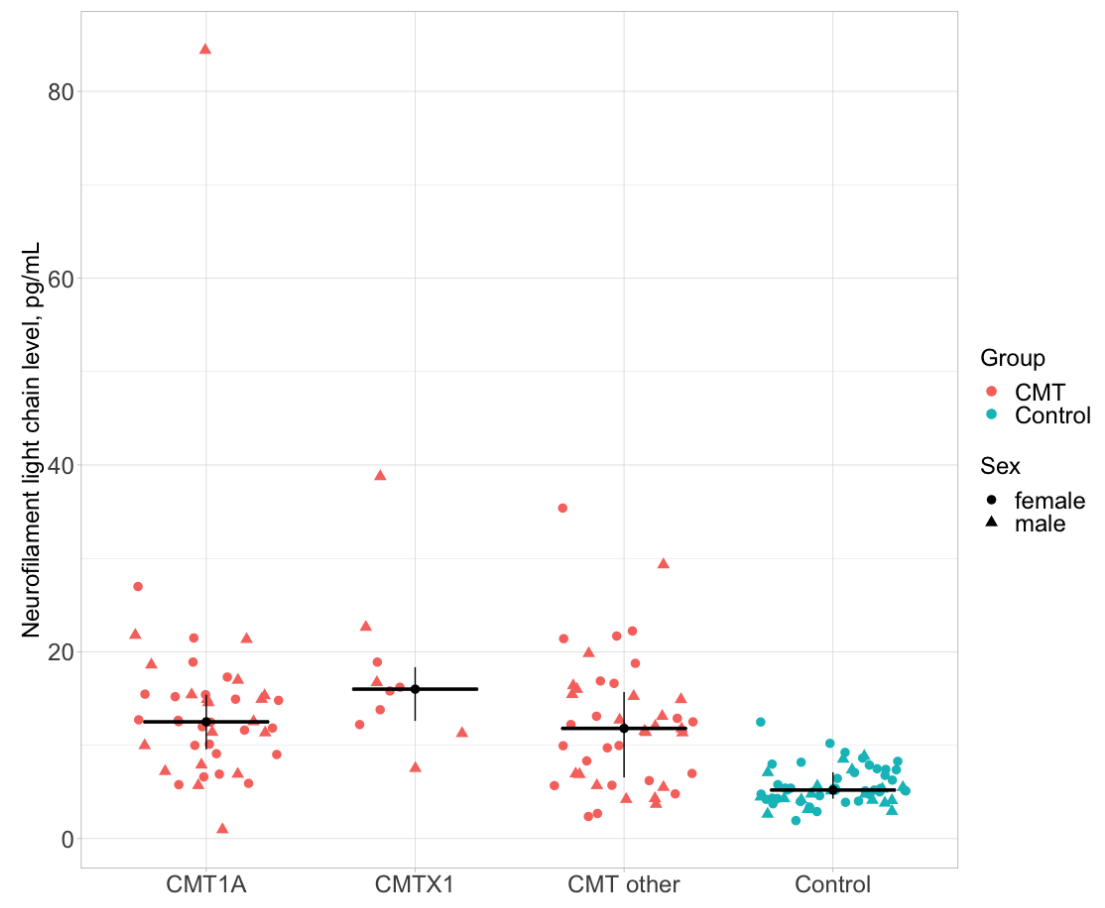

When we compared the NfL level among the different genetic groups, we found that it was significantly higher in the CMTX1 group than in the two other CMT groups ( $\mathrm{p}=0.0498)$ (Table 1 and Figure 2), despite the fact that the CMTX1 group subjects did not have the highest CMTNS. Although CMTX1 is X-linked and usually more severe in males, in our CMTX1 group there was no significant difference in the CMTNS between males and females $(p=0.222)$, nor in the level of NfL $(p=0.841)$.

To assess the correlation of NfL level with disease severity, we analysed the association with total CMTNS (which includes neurographic as well as functional features) and CMTES. The NfL concentration showed a significant but weak correlation with the CMTNS $\left(\mathrm{r}_{\mathrm{s}}=0.25, \mathrm{p}=0.012\right)$ (Figure 3). The median CMTNS and IQR for the different groups are shown in Table 1 . As the CMTES almost perfectly correlated with the CMTNS $\left(r_{s}=0.92\right.$, $\mathrm{p}<0.001)$, the NfL concentration also weakly correlated with the CMTES $\left(r_{s}=0.24, p=0.016\right)$.

Although patient-reported duration of symptoms had a moderate correlation with age $(\mathrm{r}=0.65, \mathrm{p}<0.001)$, it had a weak correlation with the CMTNS $\left(\mathrm{r}_{\mathrm{s}}=0.28, \mathrm{p}=0.006\right)$ and was not associated with the NfL level $\left(r_{s}=0.15, p=0.16\right)$.

Figure 3. Correlation of neurofilament light chain (NfL) level with CMTNS.

This article is protected by copyright. All rights reserved 


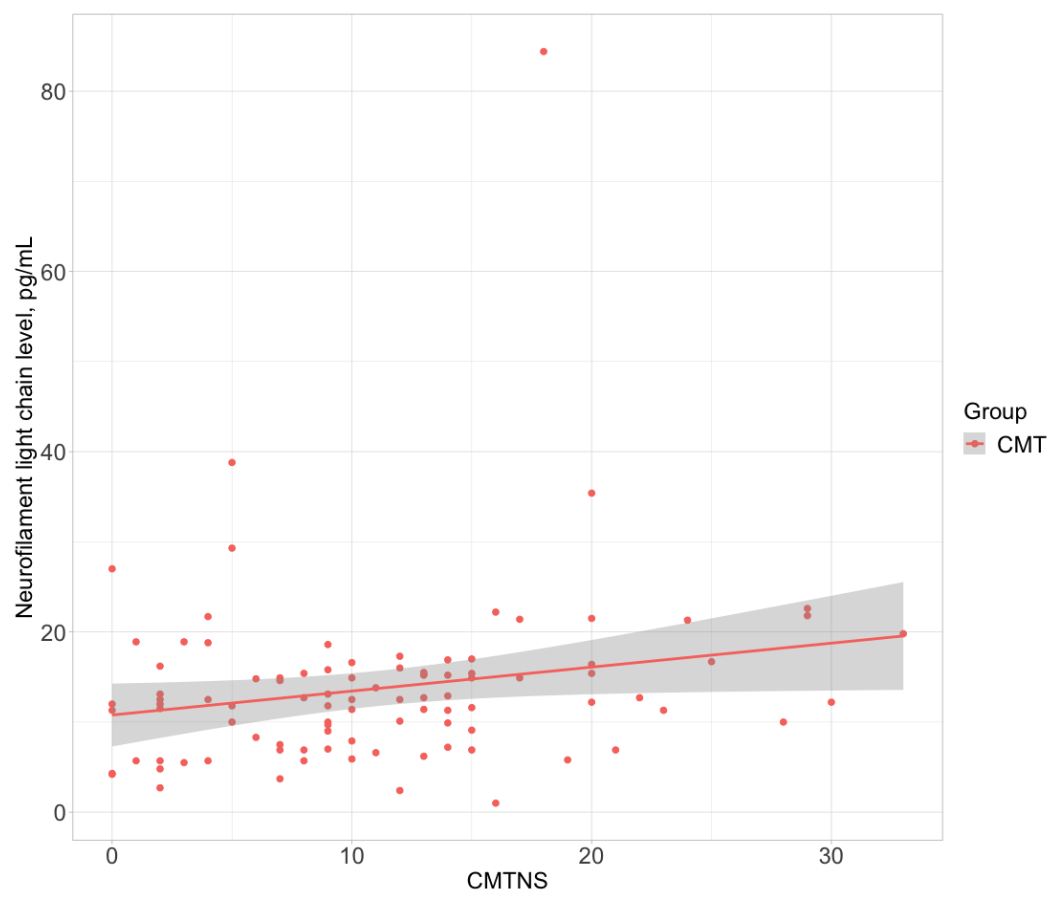

ROC analysis showed that the NfL level could be used to discriminate CMT patients from controls, with an AUC of 0.881 (95\% CI: 0.83-0.93) (Figure 4).

Figure 4. Specificity and sensitivity for plasma neurofilament light chain (NfL) level as a biomarker for CMT. 


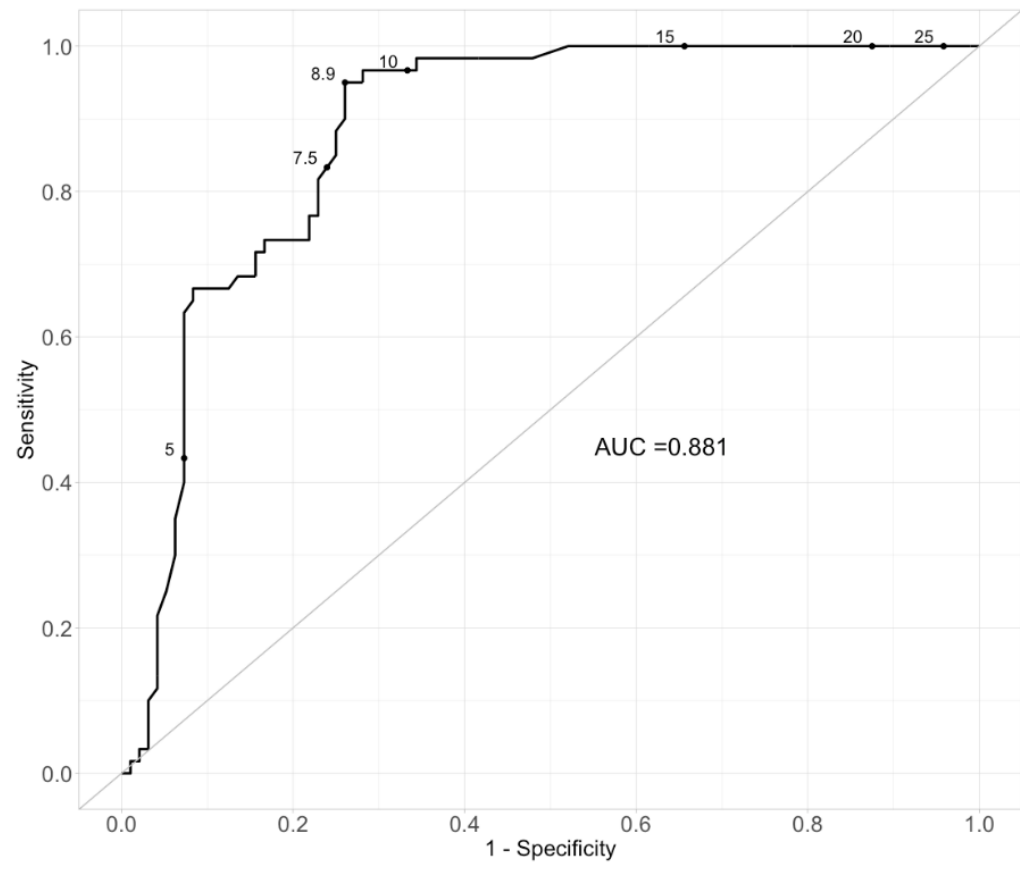

AUC - area under the curve

Evaluation of the optimal plasma NfL cut-off values (Table 2) showed that $8.9 \mathrm{pg} / \mathrm{mL}$ identified CMT patients with the highest accuracy (sensitivity of $74 \%$ and specificity of $95 \%)$.

Table 2. Sensitivity and specificity for different neurofilament light chain (NfL) threshold levels

\begin{tabular}{ccc}
\hline NfL threshold level $(\mathrm{pg} / \mathrm{mL})$ & Sensitivity $(\%)$ & Specificity (\%) \\
\hline$\geq 5$ & 92.7 & 43.3 \\
\hline$\geq 7.5$ & 76.0 & 83.3 \\
\hline$\geq 8.9$ & 74.0 & 95.0 \\
\hline$\geq 10$ & 66.7 & 96.7 \\
\hline$\geq 12.5$ & 47.9 & 98.3
\end{tabular}

\section{DISCUSSION}

The present study constitutes a validation of the plasma concentration of $\mathrm{NfL}$ as a potential disease and progression biomarker for CMT. We recruited a large group of highly phenotyped CMT neuropathy patients and healthy controls to evaluate its possible use in clinical practice and future trials.

Sandelius et al. ${ }^{12}$ recently reported that the NfL level in plasma is associated with disease severity in patients with CMT, thus raising the possibility that it could be used to 
measure disease severity and progression. However, until now, their findings have not been validated and so the next step in implementing plasma $\mathrm{NfL}$ level into real-life clinical practice has yet to be taken. To reduce any variability from the study conducted by Sandelius et al., our study assessed patients and controls in similar settings and employed the same NfL detection method that was performed in the same laboratory as described previously ${ }^{12}$. Moreover, our study included more patients with more detailed phenotypes: the CMTNS was available for a substantially higher number of patients than in Sandelius et al.'s study ( $\mathrm{n}_{\text {with }}$ CMTNS $/ \mathrm{n}_{\text {total }}$ : $96 / 96$ vs. $30 / 75$, respectively).

Our study confirmed that the plasma NfL concentration is significantly higher in CMT patients than in controls. Similar to the previous results ${ }^{12}$, our data show that CMT patients can be accurately discriminated from controls by using plasma NfL concentrations. In our study, the AUC for discriminating CMT patients from controls is 0.881 , but in the previous study a much lower AUC of 0.755 was reported ${ }^{12}$. However, despite the high AUC in our study, there is still an area of overlap between the CMT patients and health controls.

In Sandelius et al.'s study ${ }^{12}$, a significant correlation was found between NfL concentration and disease severity measured by CMTES and CMTNS, with a stronger correlation with CMTES $(r=0.46, p<0.0001)$ than with CMTNS $(r=0.37, p=0.044)$. We were able to confirm this association of NfL level with CMT severity; however, the association was weak (CMTNS: $\mathrm{r}_{\mathrm{s}}=0.25, \mathrm{p}=0.012$ and CMTES: $\mathrm{r}_{\mathrm{s}}=0.24, \mathrm{p}=0.016$ ). As both measures were highly correlated, we did not observe the difference between correlations with CMTES and CMTNS reported by Sandelius et al. Although both studies confirm a correlation between CMT severity (assessed by either CMTNS or CMTES) and NfL level, the correlation ranges from weak to medium. Therefore, more data are needed to establish whether the plasma level of $\mathrm{NfL}$ can genuinely be used to monitor disease progression.

The NfL level in plasma moderately correlated with age in our CMT patients, but this was also observed in the controls. This finding is not unexpected as an age-related increase in $\mathrm{NfL}$ is well documented in both healthy individuals and various disease groups ${ }^{26}$. It has been suggested that an increase in age-related $\mathrm{NfL}$ levels indicates neuronal injury at older age, especially if neurologic comorbidities are present ${ }^{4,12,27}$. In line with Sandelius et al.'s findings ${ }^{12}$, we detected no difference in the NfL levels between sexes in the CMT and control groups.

Patient-reported duration of symptoms moderately correlated with age, weakly correlated with disease severity (assessed with CMTNS) and did not correlate with NfL level. This may be explained by the fact that in inherited diseases, a patient's age reflects the 
duration of the disease. Furthermore, patients' perceptions of the onset of clinical symptoms may differ and patients may not remember the exact onset of their first symptoms.

Interestingly, we found that CMTX1 patients had higher NfL plasma levels in comparison to the other CMT subgroups studied. This difference was not related to sex or disease severity (measured by CMTNS). We hypothesise that the increased NfL level may have arisen from neuronal degeneration in the central nervous system - a feature previously reported amongst CMTX1 patients ${ }^{28}$. Although our CMTX1 patients did not have any signs of central nervous system involvement upon clinical examination, neuronal damage may have been subclinical. However, it could also be a false-positive finding as the size of the CMTX1 group was limited in our study $(\mathrm{n}=10)$ and Sandelius et al.'s study ${ }^{12}$ with a similar CMTX1 group size $(n=11)$ failed to find such an association.

Of note, the NfL absolute concentrations in our study were lower in comparison to other published data ${ }^{4,12}$. In a study with 335 healthy individuals aged from 38.5 to 85.6 years, the median plasma NfL level was $32.30 \mathrm{pg} / \mathrm{mL}$ (IQR 23.15-43.95) ${ }^{4}$. In another study, the mean plasma NfL level in healthy controls $(n=59)$ was $17.8 \pm 6.4 \mathrm{pg} / \mathrm{mL}^{29}$. Study in Denmark proposes NfL reference intervals for healthy controls in different age groups: the age group 18-40, 41-65, and >65 years old were 2.8-9.7 ng/L, 4.6-21.4 ng/L, and 7.5-53.8 $\mathrm{ng} / \mathrm{L}$, respectively ${ }^{30}$. In our control group, with the same NfL detection method measured a median plasma NfL level of $5.2 \mathrm{pg} / \mathrm{mL}$, which is in accordance with proposed reference intervals. This may be explained by the fact that the measurements across studies from different Laboratories were not standardised against each other and certified reference materials and/or methods for global standardisation are not yet available. This highlights the necessity for the standardisation of the test and cut-off values amongst laboratories before it can be reliably used in clinical practice.

One of our CMT1A patients (61-year-old male) had a markedly increased plasma NfL level $(84.4 \mathrm{pg} / \mathrm{mL})$ and clinically moderate phenotype (CMTNS 18). Coexistence of chronic inflammatory demyelinating polyneuropathy (CIDP) in this patient was suspected. Cooccurrence of an acquired neuropathy (e.g. CIDP) in patients with inherited neuropathies has been observed in multiple patients ${ }^{31-33}$. Because of this overlap, it is difficult to diagnose such cases. One outlier amongst the CMT cases was also reported in Sandelius et al.'s study ${ }^{12}$, but no cause for the increased level was found.

Our study shows that the NfL measurement is a promising biomarker in CMT. However, future use of NfL as a biomarker (either individually or in combination with other biomarkers) requires several issues to be addressed first: there is an area of NfL level overlap 
between CMT patients and controls; standardisation of the method and cut-off values crucial for its use in clinical settings - is lacking; NfL's non-specificity as it is elevated in various neurological disorders; and its generally modest correlation with disease severity. Furthermore, it is not known whether NfL level changes over time can be used to monitor disease progression in the short- and/or long-term. It is important to acknowledge that longitudinal data assessment is needed to evaluate $\mathrm{Nfl}$ assay capabilities for monitoring disease progression not only to reflect neuronal damage in. In Sandelius et al.'s study ${ }^{12}$, only a limited number of patients were assessed at multiple time points, thus precluding the progression of the disease to be monitored. We plan to conduct two follow-up meetings (after two and five years) for our group of patients with repeated NfL measurements and clinical evaluations which will provide additional information about the use of $\mathrm{NfL}$ in clinical practice and research. Finally, the use of NfL to identify and discriminate patients with two co-occurring conditions requires further evaluation and validation.

\section{References}

1. Morena J, Gupta A, Hoyle JC. Charcot-Marie-Tooth: From Molecules to Therapy. Int J Mol Sci. 2019;20(14):3419. doi:10.3390/ijms20143419

2. Yuan A, Rao M V, Veeranna, Nixon RA. Neurofilaments and Neurofilament Proteins in Health and Disease. Cold Spring Harb Perspect Biol. 2017;9(4):a018309. doi:10.1101/cshperspect.a018309

3. Khalil M, Teunissen CE, Otto M, et al. Neurofilaments as biomarkers in neurological disorders. Nat Rev Neurol. 2018;14(10):577-589. doi:10.1038/s41582-018-0058-z

4. Khalil M, Pirpamer L, Hofer E, et al. Serum neurofilament light levels in normal aging and their association with morphologic brain changes. Nat Commun. 2020;11(1):812. doi:10.1038/s41467-020-14612-6

5. Bergman J, Dring A, Zetterberg H, et al. Neurofilament light in CSF and serum is a sensitive marker for axonal white matter injury in MS. Neurol Neuroimmunol neuroinflammation. 2016;3(5):e271. doi:10.1212/NXI.0000000000000271

6. Preische O, Schultz SA, Apel A, et al. Serum neurofilament dynamics predicts neurodegeneration and clinical progression in presymptomatic Alzheimer's disease. Nat Med. 2019;25(2):277-283. doi:10.1038/s41591-018-0304-3 
7. Sánchez-Valle R, Heslegrave A, Foiani MS, et al. Serum neurofilament light levels correlate with severity measures and neurodegeneration markers in autosomal dominant Altheimer's disease. Alzheimers Res Ther. 2018;10(1):113. doi:10.1186/s13195-018-0439-y

8. Rohrer JD, Woollacott IOC, Dick KM, et al. Serum neurofilament light chain protein is a measure of disease intensity in frontotemporal dementia. Neurology. 2016;87(13):1329 LP - 1336. doi:10.1212/WNL.0000000000003154

9. Wilke C, Rattay TW, Hengel H, et al. Serum neurofilament light chain is increased in hereditary spastic paraplegias. Ann Clin Transl Neurol. 2018;5(7):876-882. doi:10.1002/acn3.583

10. Rostgaard N, Roos P, Portelius E, et al. CSF neurofilament light concentration is increased in presymptomatic CHMP2B mutation carriers. Neurology. 2018;90(2):e157-e163. doi:10.1212/WNL.0000000000004799

11. Mariotto S, Farinazzo A, Magliozzi R, Alberti D, Monaco S, Ferrari S. Serum and cerebrospinal neurofilament light chain levels in patients with acquired peripheral neuropathies. J Peripher Nerv Syst. 2018;23(3):174-177. doi:10.1111/jns.12279

12. Sandelius $\AA$, Zetterberg H, Blennow K, et al. Plasma neurofilament light chain concentration in the inherited peripheral neuropathies. Neurology. 2018;90(6):e518e524. doi:10.1212/WNL.0000000000004932

13. Maia LF, Maceski A, Conceição I, et al. Plasma neurofilament light chain: an early biomarker for hereditary ATTR amyloid polyneuropathy. Amyloid. 2020;27(2):97-102. doi:10.1080/13506129.2019.1708716

14. Young P, De Jonghe P, Stögbauer F, Butterfass-Bahloul T. Treatment for CharcotMarie-Tooth disease. Cochrane database Syst Rev. 2008;2008(1):CD006052CD006052. doi:10.1002/14651858.CD006052.pub2

15. Gess B, Baets J, De Jonghe P, Reilly MM, Pareyson D, Young P. Ascorbic acid for the treatment of Charcot-Marie-Tooth disease. Cochrane database Syst Rev . 2015;2015(12):CD011952-CD011952. doi:10.1002/14651858.CD011952

16. Pareyson D, Reilly MM, Schenone A, et al. Ascorbic acid in Charcot-Marie-Tooth disease type 1A (CMT-TRIAAL and CMT-TRAUK): a double-blind randomised trial. 
Lancet Neurol. 2011;10(4):320-328. doi:10.1016/S1474-4422(11)70025-4

17. Sadjadi R, Reilly MM, Shy ME, et al. Psychometrics evaluation of Charcot-MarieTooth Neuropathy Score (CMTNSv2) second version, using Rasch analysis. $J$ Peripher Nerv Syst. 2014;19(3):192-196. doi:10.1111/jns.12084

18. Murphy SM, Herrmann DN, McDermott MP, et al. Reliability of the CMT neuropathy score (second version) in Charcot-Marie-Tooth disease. J Peripher Nerv Syst. 2011;16(3):191-198. doi:10.1111/j.1529-8027.2011.00350.x

19. Tomaselli PJ, Rossor AM, Horga A, et al. Mutations in noncoding regions of GJB1 are a major cause of X-linked CMT. Neurology. 2017;88(15):1445-1453. doi:10.1212/WNL.0000000000003819

20. Richards S, Aziz N, Bale S, et al. Standards and guidelines for the interpretation of sequence variants: a joint consensus recommendation of the American College of Medical Genetics and Genomics and the Association for Molecular Pathology. Genet Med. 2015;17(5):405-424. doi:10.1038/gim.2015.30

21. R Core Team, R Foundation for Statistical Computing. R: A language and environment for statistical computing. Published online 2013.

22. Diedenhofen B, Musch J. cocor: A Comprehensive Solution for the Statistical Comparison of Correlations. PLoS One. 2015;10(4):e0121945. https://doi.org/10.1371/journal.pone.0121945

23. Robin X, Turck N, Hainard A, et al. pROC: an open-source package for R and S+ to analyze and compare ROC curves. BMC Bioinformatics. 2011;12(1):77. doi:10.1186/1471-2105-12-77

24. Ogden TL. Handling results below the level of detection. Ann Occup Hyg. 2010;54(3):255-256. doi:10.1093/annhyg/mep099

25. Huston C, Juarez-Colunga E. Guidelines for Computing Summary Statistics for DataSets Containing Non-Detects.; 2009.

26. Gaetani L, Blennow K, Calabresi P, Di Filippo M, Parnetti L, Zetterberg H. Neurofilament light chain as a biomarker in neurological disorders. J Neurol Neurosurg Psychiatry. 2019;90(8):870-881. doi:10.1136/jnnp-2018-320106 
27. Bridel C, van Wieringen WN, Zetterberg H, Tijms BM, Teunissen CE, Group and the NFL. Diagnostic Value of Cerebrospinal Fluid Neurofilament Light Protein in Neurology: A Systematic Review and Meta-analysis. JAMA Neurol. 2019;76(9):10351048. doi:10.1001/jamaneurol.2019.1534

28. Abrams C. GJB1 Disorders: Charcot Marie Tooth Neuropathy (CMT1X) and Central Nervous System Phenotypes. (Adam M, Ardinger H, Pagon R, Al. E, eds.). www.ncbi.nlm.nih.gov/books/NBK1374/

29. Lin Y-S, Lee W-J, Wang S-J, Fuh J-L. Levels of plasma neurofilament light chain and cognitive function in patients with Alzheimer or Parkinson disease. Sci Rep. 2018;8(1):17368. doi:10.1038/s41598-018-35766-w

30. Hviid CVB, Knudsen CS, Parkner T. Reference interval and preanalytical properties of serum neurofilament light chain in Scandinavian adults. Scand J Clin Lab Invest. 2020;80(4):291-295. doi:10.1080/00365513.2020.1730434

31. Shimizu K, Hanajima R, Shimizu T, et al. Coexistence of Charcot-Marie-Tooth disease type $1 \mathrm{~A}$ and chronic inflammatory demyelinating polyradiculoneuropathy with conduction blocks. Neurol Clin Neurosci. 2016;4(5):192-194. doi:10.1111/ncn3.12071

32. Ginsberg L, Malik O, Kenton AR, et al. Coexistent hereditary and inflammatory neuropathy. Brain. 2004;127(Pt 1):193-202. doi:10.1093/brain/awh017

33. Vital A, Vital C, Lagueny A, et al. Inflammatory demyelination in a patient with CMT1A. Muscle Nerve. 2003;28(3):373-376. doi:10.1002/mus.10404

This article is protected by copyright. All rights reserved 


\section{THE IMPORTANCE OF GREY AND WHITE MATTER In Multiple Sclerosis}

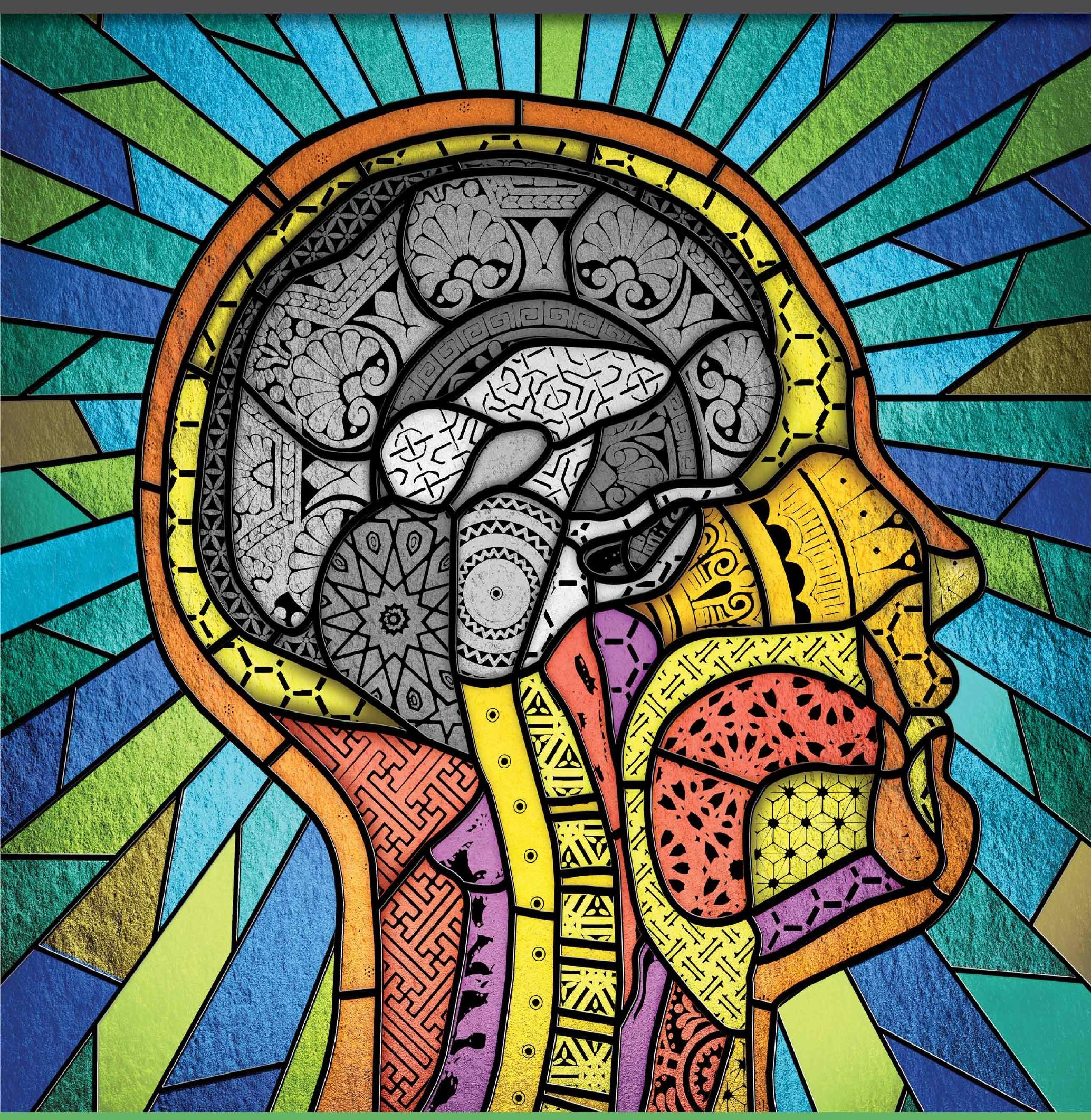

Visit GreyAndWhiteMS.com for more information. 